\title{
Der Radiologe als Gatekeeper
}

\author{
Subspezialisierung in klinische Fächer beim ECR präsent.
}

Mehr intelligente Technologie und Roboter werden in der Zukunft auch in der Medizin weniger Platz für menschliche Fehler und eine bessere Diagnose zur Folge haben. Die Rolle des Radiologen in diesem Zukunftsszenario ist für András Palkó, Vorstand der radiologischen Abteilung der Universität Szeged und Präsident der Europäischen Radiologie Gesellschaft (SR) klar: „Er wird seine Expertise als Gatekeeper einbringen und ist Teil des interdisziplinären Konsultationsteams." Die neuesten Entwicklungen des Fachs Radiologie sowohl technisch als auch methodisch und vor allem im interdisziplinären Zusammenspiel stand vom 1. Bis 5. März im Mittelpunkt dies diesjährigen Europäischen Kardiologenkongresses (ECR) in Wien.

Kürzere und billigere Wege zur Diagnose werden mithilfe der Informationstechnologien wie Cloud Computing und Datami-

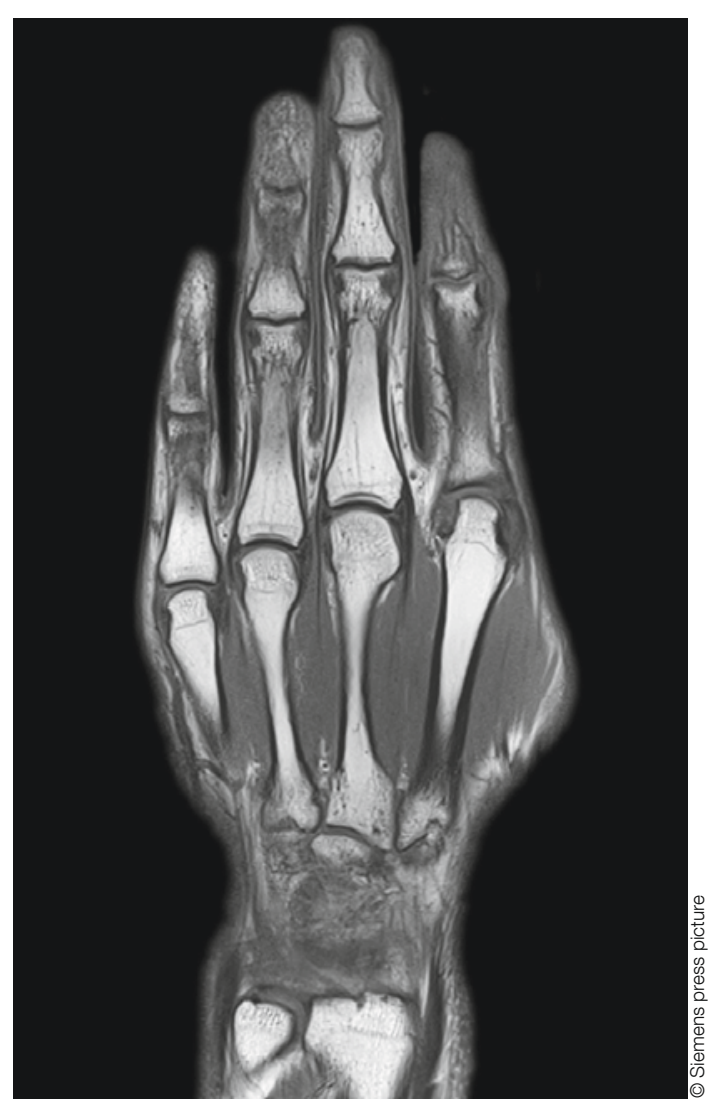

Weniger menschliche Fehler durch moderne Technologien - das ist die Hoffnung der Zukunft. ning und einer Vernetzung nationaler und internationaler Krankengeschichten-Datenbanken möglich - welche Untersuchungen schließlich am zielführendsten sind, kann der Radiologe mit klinischem Hintergrundwissen effizient entscheiden. Die Beschäftigung und Integration der neuen Rahmenbedingungen in die eigene Arbeit ist dafür ebenso notwendig, wie eine Subspezialisierung, so Palkó. Allerdings geht es dabei nicht um die technischen Modalitäten sondern um die klinischen Fächer. Dementsprechend strukturiert zeigte sich auch der ECR 2012. Einen der Schwerpunkt stellte die Bildgebung der weiblichen Brust mit den unterschiedlichen Kontroversen darüber dar.

Je mehr Informationen wir haben, desto mehr Interpretationen sind möglich, stellte Matthew G. Wallis, Cambridge University Hospitals fest. Es geht um die Balance zwischen Vor- und Nachteilen. Dazu zählt beispielsweise der Einsatz von Ultraschall im Brustkrebsscreening. „Um eine Krebserkrankung zu finden, müssen in 1.000 Frauen 100 weitergehend untersucht werden, so Wallis, weil irgendetwas gefunden wird, aber niemand weiß, ob die Konsequenz eine Bedeutung für die betroffenen Frau hat." Fest stehe, so Wallis, dass beispielsweis mit einem Screening für Frauen unter 50 Jahren mehr Brusterkrankungen diagnostiziert werden, allerdings würde dadurch auch die Zahl falsch positiver Befunde deutlich erhöht werden. Ein Screening von Frauen zwischen 40 und 50 Jahren reduziert die Mortalität über einen Zeitraum von 25 Jahren um 19 Prozent. Das bedeutet, dass 1000 Frauen gescreent werden müssen um drei Frauen it Brustkrebs zu finden. Prod drei Patienten wird allerdings eine Frau nicht gefunden, die erkrankt ist. „Die Paranoia des Radiologen ist, jene Patientin nicht zu finden“, erklärte Wallis und stellte klar: Die behandelnden
Radiologen müssen akzeptieren, dass ihre Tätigkeit nie mit der absoluten Sicherheit verbunden ist, jede einzelne Krebserkrankung zu erkennen." Daher sei auch die Kommunikation sowohl mit der Patienten als auch mit den Kollegen ein wesentlicher Aspekt des Patientenmanagements. Neueste Technologien ermöglichen nun bereits wesentlich bessere Differenzierungen der Läsionen, allerdings sei es weiterhin nicht möglich vorherzusagen, wie sich der individuelle Tumor entwickelt, schränkte Wallis ein: Manche sind hoch aggressiv, manche entwickeln sich langsam und wenig aggressiv. Wir müssen alle behandeln."

\section{HI-Virus vier Tage nach Infektion im Gehirn}

Die Behandlung von HIV/AIDS hat sich zwar in den vergangenen zehn Jahren durch die antiretroviralen Medikamente wesentlich verbessert, mit 33,3 Millionen Infizierten weltweit im Jahr 2009 ist die Epidemie aber weiterhin nicht unter Kontrolle, stellte Univ.-Prof. Dr. Majda M. Thurnherr, Medizinische Universität Wien, fest. Allerdings haben sich in den unterschiedlichen Manifestationen im Gehirn Veränderungen ergeben, die für die Interpretation der Bildgebung von Bedeutung sind. Von den früher häufig aufgetretenen opportunistischen Infektionen mit Bakterien, Parasiten oder Viren hat sich die Infektion in Richtung steigender Zahl an neurologischen und kognitiven Störungen verschoben. Bereits vier Tage nach einer Infektion, so Thurnherr, findet sich das HI-Virus im Gehirn: „Die anitretrovirale Therapie stoppt die Hirninfektion nicht, aber sie verlangsamt sie." Manche Läsionen können auch eine Reaktion des Immunsystems auf eine antiretrovirale Therapie sein, daher sei manchmal ein Abwarten durchaus sinnvoll, um die Entwicklung richtig einschätzen zu können. Herkömmliche und fortgeschrittene MR-Techniken wie Spektroskopie, Perfusions MR, funktionelle MRT und die Diffusionsbildgebung sollten in Zukunft die möglichst frühe Erkennung neurologischer durch HIV verursachter Störungen zu ermöglichen. Aber, so Thurnherr: „Es gibt keine einzelne beste Technik der Bildgebung. Das Konzept ist immer multimodal." 